\title{
Discovery of Two Very Low-Mass Binaries: Final Results of an Adaptive Optics Survey of Nearby M6.0-M7.5 Stars
}

\author{
Nick Siegler ${ }^{1}$, Laird M. Close ${ }^{1}$, Kelle L. Cruz ${ }^{2,3}$, Eduardo L. Martín ${ }^{4}$, \& I. Neill Reid ${ }^{2,5}$
}

\begin{abstract}
We present updated results of a high-resolution, magnitude limited $\left(\mathrm{K}_{s}<\right.$ $12 \mathrm{mag}$ ) imaging survey of nearby low-mass M6.0-M7.5 field stars. The observations were carried out using adaptive optics at the Gemini North, VLT, Keck II, and Subaru telescopes. Our sample of 36 stars consists predominantly of nearby $(\lesssim 30 \mathrm{pc})$ field stars, 5 of which we have resolved as binaries. Two of the binary systems, 2MASSI J0429184-312356 and 2MASSI J1847034+552243, are presented here for the first time. All 5 discovered binary systems have separations between $00^{\prime \prime} 08-0$ - .53 (2-9 AU) with similar mass ratios ( $q>0.8$, $\left.\Delta \mathrm{K}_{s}<1 \mathrm{mag}\right)$. This result supports the hypothesis that wide (a $>20 \mathrm{AU}$ ) very low-mass $\left(\mathrm{M}_{t o t}<0.19 \mathrm{M}_{\odot}\right)$ binary systems are rare. The projected semimajor axis distribution of these systems peak at $\sim 5 \mathrm{AU}$ and we report a sensitivitycorrected binary fraction of $9_{-3}^{+4} \%$ for stars with primaries of spectral type M6.0M7.5 with separations $\gtrsim 3 \mathrm{AU}$ and mass ratios $\mathrm{q} \gtrsim 0.6$. Within these instrumental sensitivities, these results support the overall trend that both the semimajor axis distribution and binary fraction are a function of the mass of the primary star and decrease with decreasing primary mass. These observations provide important constraints for low-mass binary star formation theories.
\end{abstract}

Subject headings: instrumentation: adaptive optics - binaries: general-stars: low mass - stars: individual ()

\footnotetext{
${ }^{1}$ Steward Observatory, University of Arizona, 933 N. Cherry Ave., Tucson, AZ 85721, USA

${ }^{2}$ Department of Physics and Astronomy, University of Pennsylvania, 209 South 33rd Street, Philadelphia, PA 19104, USA

${ }^{3}$ American Museum of Natural History, Department of Astrophysics, Central Park West at 79th St., New York, NY, 10023 USA

${ }^{4}$ Instituto de Astrofisica de Canarias, La Laguna, Tenerife, E-38200 Spain

${ }^{5}$ Space Telescope Science Institute, 3700 San Martin Drive, Baltimore, MD 21218, USA
} 


\section{Introduction}

One of the main motivations for measuring the binary fraction of stars is to better understand the process of star formation itself. After all, stars like our own Sun are preferentially produced in multiple systems (Duquennoy \& Mayor 1991). The classic stellar formation mechanism of molecular cloud core collapse and fragmentation, however, has a hard time explaining the tightest systems. While this mechanism can explain wide binary systems (semimajor axis $\gtrsim 10 \mathrm{AU}$ ), it has some difficulties explaining the formation of tight systems (Bate, Bonnell, \& Bromm 2002). Additionally, the multiplicities of the lowest mass stars and brown dwarfs appear to be statistically different from those of more massive systems (Close et al. (2003) and references within). These differences, if proven to be real, provide important clues and constraints to theoretical stellar formation models.

The continuously improving statistics of binary stars brings clarity to the paradigm that the binary fraction and semimajor axis distribution are functions of the central star mass. Surveys of $\mathrm{G}$ dwarfs estimate a multiplicity fraction of approximately $50 \%$ for separations of $3 \mathrm{AU}$ and greater (Duquennoy \& Mayor 1991). Other surveys of similar sensitivity to systems wider than $3 \mathrm{AU}$ have found that early M dwarfs (M0-M4) have measured binary fractions of $\sim 32 \%$ (Fischer \& Marcy 1992) while late M/early L dwarfs (M8.0-L0.5) estimate fractions $\sim 15 \%$ (Close et al. 2003). The trend appears to continue to the coolest objects - L dwarfs reporting 10-15\% (Bouy et al. 2003; Gizis et al. 2003) and $\mathrm{T}$ dwarfs at $\sim 10 \%$ (Burgasser et al. 2003). The same surveys infer semimajor-axis separations to also be a function of primary mass. While $\mathrm{G}$ and early $\mathrm{M}$ dwarfs (M0-M4) show broad separation peaks of $\sim 30 \mathrm{AU}$, late $\mathrm{M}(\geq \mathrm{M} 8)$, L, and T dwarfs appear to have separations tightly peaked at $\sim 4$ AU (Close et al. 2003). A similar result has been shown to apply to the sequence of members in the Pleiades cluster, from solar-type stars to brown dwarfs (Martin et al. 2003). Together, these results are providing both clues and empirical constraints on star formation mechanisms as well as potentially help calibrate the mass-age-luminosity relation for very low-mass (VLM) stars.

In Siegler et al. (2003), hereafter referred to as Paper I, we presented results from the largest flux limited $\left(\mathrm{K}_{s}<12 \mathrm{mag}\right)$ survey of nearby field M6.0-M7.5 dwarfs. The binary fraction of this narrowly defined spectral type range had not been quantified as those of stars slightly earlier (M0-M4; Fischer \& Marcy 1992) and later (M8-L0.5; Close et al. 2003). Considering the differences in binary charactersitics as a function of mass as discussed above, would M6.0-M7.5 binaries have intermediary characteristics to their main sequence neighbors or would they resemble the ultracool dwarfs?

Paper I's sample consisted of 30 stars and presented the discovery of three new binary systems using the University of Hawaii visitor AO system Hokupa'a (Graves et al. 1998) at 
the Gemini North telescope. The discoveries followed characteristics of other VLM binary systems, namely relatively equal mass components $(q>0.8)$ with projected separations less than $16 \mathrm{AU}$. In this paper we present our two latest binary discoveries from this spectral range, 2MASSI J0429184-312356 and 2MASSI J1847034+552243, hereafter referred to as 2M 0429 and 2M 1847. These binaries were discovered with the VLT and the Subaru AO facilities, respectively. The total M6.0-M7.5 sample size is increased to 36 and we update the binary fraction results with those presented in Paper I. We present our observations and results in the following section and examine the systems' derived characteristics such as distance, age, temperature, spectral type, and mass in $\S 3$. In $\S 4$ we conclude by discussing the binary frequency and separation distribution of M6.0-M7.5 dwarfs.

\section{Observations and Results}

\subsection{The Sample}

We selected a flux-limited sample of 36 objects consisting of M6.0-M7.5 dwarfs with $\mathrm{K}_{s}<12 \mathrm{mag}$ and $\mathrm{J}_{-} \mathrm{K}_{s}>0.95 \mathrm{mag}$ from mainly 2MASS stars listed in Cruz et al. (2003), Reid et al. (2002), and Gizis et al. (2000). Paper I discusses the first 30 observations and we report here the most recent 6 . One of the 6 targets is a recently discovered high proper motion M dwarf - SO 025300.5+165258 ( 3.6 pc away; Teegarden et al. 2003). We discuss this star further in $§ 2.5$. We also note that we observed at Subaru on 2003 July 10 (UT) another recently-discovered high proper motion M dwarf 2MASSI J1835379+325954 ( 5.7 pc away; Reid et al. 2003). While not part of this sample due to its later spectral type (M8), it was observed at high resolution and found to have no $q>0.8$ companions at separations $>0{ }^{\prime \prime} 1$.

\subsection{The Telescopes and their AO Systems}

The 30 targets from Paper I were all observed at the Gemini North telescope. The 6 targets presented here were observed at the Subaru and VLT Observatories. Due to its recent discovery and proximity, we conducted additional long integrations of SO $025300.5+165258$

with the Keck II telescope. Interestingly, the AO systems at these telescopes represent the three major wavefront sensor (WFS) technologies currently in use today. Gemini North, at the time of our observations, and Subaru, currently, use 36-element curvature WFSs, the VLT has an infra-red Shack-Hartman, and the Keck II utilizes a visible Shack-Hartman. This survey provided the opportunity to compare and contrast how different AO WFSs differ in their abilities to lock on faint targets. 
As discussed in detail in Paper I, one of the challenges in utilizing AO is locating sufficiently bright guide stars near enough one's science objects to minimize uncertainty in the image quality introduced by isoplanicity. This is best achieved when using the target object itself as the AO guide star. This shifts the criterion of target selection from the availability of bright natural guide stars $(\mathrm{R} \lesssim 15 \mathrm{mag})$ to the sensitivity of the respective telescope's AO system, in particular the WFS. This becomes quite important because the probability of locating a $\mathrm{R}=15 \mathrm{mag}$ star within $30^{\prime \prime}$ of one's science target is only about $\sim 15 \%$ (Fig. 3.10; Roddier 1999). The ability to guide on fainter stars also allows for both larger sample sizes and improved contrast ratios.

We were able to observe the faintest of our targets (V 19.0-19.5 mag, I 15.5-16 mag) only with the former Gemini North AO system Hokupa'a where we conducted the majority of the observations. No other current AO system can lock onto such faint targets. Hokupa'a, decommisioned in 2003, was a curvature-based AO system which employed in its WFS red-sensitive, photon-counting avalanche photodiodes with effectively zero read-noise. Consequently, this type of sensor is ideally suited for guiding on intrinsically faint objects as long as they are relatively red ( $\mathrm{V}-\mathrm{I} \sim 4 \mathrm{mag})$. The Keck II telescope with a more traditional Shack-Hartman WFS allows for improved angular resolution with higher obtainable Strehl ratios but requires brighter targets $(\mathrm{V} \sim 15 \mathrm{mag})$. We compare and contrast the performance of these two types of WFS technologies in Siegler, Close, \& Freed (2002). At both Subaru and the VLT we were able to lock on our faint low mass targets with $\mathrm{I} \lesssim 15.2 \mathrm{mag}$ $\left(\mathrm{K}_{s} \lesssim 11.2 \mathrm{mag}\right)$

\subsection{Observations}

The two discovered binary systems, 2M 0429 and 2M 1847, were detected at the VLT and Subaru observatories on 2003 February 13 (UT) and 2003 July 10 (UT), respectively. A total of 6 dwarfs from our sample were observed during these two runs. Table 1 lists the 4 low-mass dwarfs observed with no likely physical companion detections between $\sim 0{ }^{\prime}$. 1 $15^{\prime \prime}$. For completeness we also include the 27 single stars observed in Paper I. Table 2 lists the observable properties of the 2 new binary systems along with the 3 systems presented in Paper I. Target stars were considered "observed" when a minimum corrected FWHM of $\sim 0$ '.15 in $\mathrm{H}$ band was achieved.

Each of the observations were made by dithering over 4 different quadrant positions on the infrared camera detector. For all targets we obtained both unsaturated $\mathrm{H}$ or $\mathrm{K}_{s}$ images ( $\leq 10 \mathrm{~s}$, "short" images), depending on seeing conditions, and saturated H images (30s, "deep" images) to gain sensitivity to potential faint companions. 
At Subaru we used the Coronagraphic Imager with AO (CIAO) without using the coronagraphic mask feature. The detector is a $1024 \times 1024$ ALADDIN II InSb infrared hybrid array with a platescale of $0^{\prime \prime} 0217$ pixel $^{-1}$ (Tamura et al. 2000). For 2M 1847 we took a total of $12 \times 10 \mathrm{~s}$ short exposures at $\mathrm{H}$ and $\mathrm{K}_{s}, 12 \times 20 \mathrm{~s}$ at $\mathrm{J}$, and $12 \times 60 \mathrm{~s}$ deep exposures at $\mathrm{H}$. At the VLT we observed with the Nasmyth AO System/NIR Imager and Spectrograph (NACO) system on UT4 (Yepun) which contains a 1024×1024 ALADDIN II InSb infrared hybrid array detector with a platescale of $0^{\prime \prime} 0271$ pixel $^{-1}$. NACO is unique in that it utilizes an infrared WFS. We found that the infrared WFS was most efficient for objects with $\mathrm{K}_{s} \leq 11.2 \mathrm{mag}$. For $2 \mathrm{M} 0429$ we took a total of $16 \times 0.5 \mathrm{~s}$ short exposures at $\mathrm{H}, 8 \times 0.5 \mathrm{~s}$ at $\mathrm{K}_{s}, 12 \times 1 \mathrm{~s}$ at $\mathrm{J}$, and $12 \times 30$ s deep $\mathrm{H}$ frames.

\subsection{Reduction}

The images were reduced using an AO data reduction pipeline written in the IRAF language as first described in Close et al. (2002a). The pipeline produces final unsaturated exposures in $\mathrm{J}, \mathrm{H}$, and $\mathrm{K}_{s}$ with deep $720 \mathrm{~s}$ exposures at $\mathrm{H}$ band for each observed binary system. The dithering of the shorter exposures produces a final $30^{\prime \prime} \times 30^{\prime \prime}$ image with a high $\mathrm{S} / \mathrm{N}$ region in a $10^{\prime \prime} \times 10^{\prime \prime}$ box centered on the binary. In order to detect close companions within $1^{\prime \prime}$ of the central star we filter out the low spatial frequency components of the deep images leaving behind high frequency residuals in the PSF (unsharp masking). No faint companions, however, were found within the halo of our central stars using this technique. Both binary systems were detected from reductions of the shorter exposures. Figures 1 and 2 show $\mathrm{K}_{s}$ images of the two new systems.

Photometry for the more widely separated 2M 0429 was performed using the DAOPHOT PSF fitting photometry package in IRAF. The PSFs used were unsaturated single stars observed during the same night with similar IR brightness, spectral type, and air mass. The errors in $\Delta$ mag, listed in Table 2 , are the differences in the photometry between 2 similar PSF stars.

DAOPHOT could not successfully separate the strongly blended components of 2M 1847 due to lower Strehl ratios caused by observing through a 1.4 airmass (the Strehl ratio and hence resolution were better at airmass of 1 when the binary was initially discovered and its components more clearly separated, however, technical difficulties resulted in delayed image acquisition). Consequently, we remove the low spatial frequencies of the binary revealing their high-frequency cores. We then perform aperture photometry using IRAF PHOT. This purely differential technique preserves the relative magnitude difference between each component while removing sufficient primary halo flux to reveal the companion centroids in $\mathrm{K}_{s}$. 
The technique gave reliable $\Delta$ mags and was verified on binary images with known $\Delta$ mags.

We calculate individual fluxes and their uncertainties from the measured binary flux ratios and the integrated 2MASS apparent magnitudes (2MASS All-Sky Point Source Catalog), along with their respective uncertainties. Table 3 lists the photometry and derived characteristics of the new binary systems.

\subsection{An Example of Sensitivity: The Special Case of 2MASS 0253}

One of our targets observed to have no stellar companions deserves special mention. $2 \mathrm{M} 02530084+1652532$ (hereafter 2M 0253) is a newly discovered M6.5 dwarf (Teegarden et al. 2003) remarkably only $3.6 \mathrm{pc}$ away. It was discovered from a search of the SkyMorph database of the Near Earth Asteroid Tracking (NEAT) project (Pravdo et al. 1999) as a high proper motion object $\left(5^{\prime \prime} \mathrm{yr}^{-1}\right)$. The star's proximity presented a rare observational window for the direct imaging of several Jupiter-mass, extrasolar planets. We were able to both probe semimajor axis separations to within $\sim 3 \mathrm{AU}$ of the star, comparable to the separations of known extrasolar planets detected through radial velocity studies $(\mathrm{a} \lesssim 6 \mathrm{AU}$; http://exoplanets.org) and outside the speckle-dominated region on the detector ( $\left.\gtrsim 1^{\prime \prime}\right)$. We were the first to observe this object with high resolution on 2003 July 14 (UT) using the NIRC2 camera and AO (Wizinowich et al. 2000) on the W. M. Keck II telescope. The $0^{\prime \prime} 01$ pixel $^{-1}$ plate scale mode was used on the $1024 \times 1024$ pixel array.

2M 0253 was only observable for approximately 1 hour in the early morning. We achieved sensitivity to companions of $\mathrm{H}=19.6$ at $1^{\prime \prime} .5$ in 24 min of total integration time $(49 \times 30$ s frames in a 4-dither pattern). We fully saturated the central star so as to allow for the detection of any massive faint Jupiter planets orbiting $\gtrsim 1^{\prime \prime}(>2.6 \mathrm{AU})$ from the central star. No faint companions were detected.

The 24 min of total integration time enables us to establish upper limits on planetary masses orbiting this star. We construct an unsaturated PSF of the star by replacing the saturated core with scaled unsaturated pixels from a short exposure. We determine maximum $\mathrm{H}$ band $\Delta$ mag contrasts by combining scaled models of faint companions (with appropriate PSFs) at various radial distances until a $5 \sigma$ detection is obtained. Figure 3 shows the resulting $5 \sigma$ limiting magnitudes at several radial distances from the central star. The horizontal dashed lines indicate the $\mathrm{H}$ band $\Delta$ mag required for the detection of $5 \mathrm{Gyr}, 10 \mathrm{M}_{J}$ and $25 \mathrm{M}_{J}$ objects using the models of Burrows, Sudarsky, \& Lunine (2003). We use the

peak of their $\mathrm{H}$ band spectra to estimate the flux emission in this exercise. The star's age is not known but based on its high tangential velocity we can assume it is an older object 
(Wielen 1974). The figure demonstrates sensitivity to an 11 Jupiter-mass extrasolar planet at only $\sim 4 \mathrm{AU}\left(1.5^{\prime \prime}\right)$ away. In Figure 4 we show the fully reduced Keck image of 2M 0253 spatially filtered of its low frequency halo, leaving behind high frequency residuals in the core (superspeckles). This image also illustrates that with conventional AO, speckle noise limits the detection of faint companions within the inner $\sim 1^{\prime \prime}$ of the halo.

\section{Analysis}

\subsection{Are the Companions Physically Related to the Primaries?}

From the total of 69 objects already observed in both this survey and a companion survey of M8.0-L0.5 stars by the authors (Close et al. 2003), we did not detect any additional unknown red $\left(\mathrm{J}-\mathrm{K}_{s}>0.8 \mathrm{mag}\right)$ background objects in $6.2 \times 10^{4}$ square arcsec. Therefore, we estimate the probability of a chance projection of a comparably red object within $0.5^{\prime \prime}$ of the primary to be $<1.3 \times 10^{-5}$. As we argued in Paper I, with an M6-M8 dwarf density of $0.007 \mathrm{pc}^{-3}$ in the local solar neighborhood (Reid \& Gizis 1997a), the probability of an apparent companion being just a background star at, for example, twice the distance of the target star (hence fainter by a $\Delta$ magnitude of $1.5 \mathrm{mag}$ ) and appearing within $0.5^{\prime \prime}$ of any of our targets is estimated to only be $\sim 3 \times 10^{-7}$. Additionally, none of the companion images appear spatially extended as might be expected of background galaxies. Therefore, we conclude that both of the very red companions are physically associated with their primaries and hereafter we will refer to them as 2M 0429B and 2M 1847B.

\subsection{Distances}

Neither of the 2 binary systems have published trigonometric parallaxes. We estimate distances to both primaries from a color-magnitude diagram developed in Paper I based on trigonometric parallaxes of other well-studied, late-M, field dwarfs from Dahn et al. (2002). Using corresponding 2MASS photometry for each star with a trigonometric parallax, we estimated a linear least-squares fit of $\mathrm{M}_{K_{s}}=7.65+2.13\left(\mathrm{~J}-\mathrm{K}_{s}\right)$ for the spectral range M6.5L1. This relationship has a $1 \sigma$ error of $0.33 \mathrm{mag}$, which has been added in quadrature to the $\mathrm{J}$ and $\mathrm{K}_{s}$ photometric errors to yield the primarys' $\mathrm{M}_{K_{s}}$ values reported in Table 3 . We then use the distance modulus of the primary to estimate the distances to the binaries. The calculated distances are listed in Table 3. 


\subsection{Spectral Types and Temperatures}

We do not have spatially resolved spectra of the individual components in either of the 2 new systems. We estimate the spectral types of each of the binary components by using the relation $\mathrm{SpT}=3.54 \mathrm{M}_{K_{s}}-27.20$ derived in Paper I from the data set of Dahn et al. (2002) (eg. $\mathrm{SpT}=8$ is an $\mathrm{M} 8, \mathrm{SpT}=10$ is an L0, etc). This relationship has a $1 \sigma$ error of 0.85 spectral types which when taken in quadrature with the uncertainty in $\mathrm{M}_{K_{s}}$ gives an overall uncertainty of about 1.5 spectral types. Fortunately, none of analysis is dependent on these spectral type estimates. The results are listed in Table 3.

Effective temperatures of the binary components are estimated from the DUSTY evolutionary tracks (Chabrier et al. 2000) using calculated $\mathrm{M}_{K_{s}}$ values and estimated ages (see Figures 5 and 6). We estimate 2M0429A and 2M0429B to have effective temperatures of $2690_{-170}^{+160} \mathrm{~K}$ and $2240_{-260}^{+190} \mathrm{~K}$, respectively; $2 \mathrm{M} 1847 \mathrm{~A}$ and $2 \mathrm{M} 1847 \mathrm{~B}$ are estimated at $2760_{-260}^{+280} \mathrm{~K}$ and $2690_{-210}^{+220} \mathrm{~K}$, respectively. These estimated temperatures are in very good agreement with those predicted in Dahn et al. (2002) for the given spectral types.

\subsection{Ages and Masses}

Estimating the age of late-type field dwarfs without Li measurements or established cluster membership is difficult. Consequently, we conservatively assume a mean age of $\sim$ 5 Gyr for our objects with uncertainty spanning the range of common ages in the solar neighborhood (0.6 - 7.5 Gyr; Caloi et al. 1999).

To estimate masses of these objects we rely on luminosity-mass-age models for VLM stars and brown dwarfs. We utilize the DUSTY models to provide theoretical estimates for both stellar and substellar masses as a function of both absolute $\mathrm{K}_{s}$ magnitude and age. The tracks are calibrated for the $K_{s}$ bandpass (I. Baraffe, private communication) and we extrapolate the isochrones from 0.10 to $0.11 \mathrm{M}_{\odot}$ so as to enclose the upper mass limits of our central stars. The companion's absolute magnitude is simply determined by adding the measured $\Delta \mathrm{K}_{s}$ to its primary star's $\mathrm{M}_{K_{s}}$. The crosses in Figures 5 and 6 indicate the best estimates of where the binary components lie on the $5 \mathrm{Gyr}$ tracks and their uncertainties are represented by the shaded regions. 2M 0429A's region of uncertainty is displayed in the upper right while its companion is displayed in the lower left. Because the 2M 1847 binary system is of near equal magnitudes, their regions of uncertainty largely overlap. In this case the slightly more massive primary's region of uncertainty is indicated in bold outline and the portion of the companion's not overlapping is dashed. The maximum mass is related to the minimum $\mathrm{M}_{K_{s}}$ at the oldest possible age; the minimum mass is related to the maximum $\mathrm{M}_{K_{s}}$ 
at the youngest possible age. Table 3 lists the estimated masses for both binary systems. Both systems' primary masses are consistent with M7-type dwarfs and their secondaries are most likely stellar, however, 2M 0429B's uncertainties extend well into the substellar region according to the model. The uncertainty in the masses, as well as in the effective temperatures, is largely driven by the uncertainty in our determination of $\mathrm{M}_{K_{s}}(\sigma=0.33$ mag) as obtained from the $\left[\mathrm{M}_{K_{s}}, \mathrm{~J}-\mathrm{K}_{s}\right]$ color magnitude diagram linear fit from Paper $\mathrm{I}$. Future observations of trigonometric parallaxes would significantly reduce the uncertainty in $\mathrm{M}_{K_{s}}$ and hence the masses and temperatures.

\section{Discussion}

\subsection{The Binary Frequency of M6.0-M7.5 Stars}

We update the binary fraction statistics of M6.0-M7.5 stars combining the latest results presented here (2 binaries resolved out of 6 ) with those from Paper I (3 binaries out of 30).

This implies an observed, uncorrected binary fraction of $14_{-4}^{+8} \%$ using a Poisson distribution for the uncertainty (Burgasser et al. 2003). However, this sample was originally drawn from a magnitude-limited sample and hence the observed binary fraction is biased due to the leakage of equal magnitude binaries into our sample from further distances (Malmquist bias). We therefore need to correct our result due to this bias as well as consider sample incompleteness due to undetected very tight lower mass companions.

To compensate for the fainter single stars not included in our flux-limited sample we first adjust for a larger observed volume due to the discovered binaries by a volume correction factor. This factor is simply the ratio of the spherical volume containing $95 \%$ of our detected binaries and the spherical volume containing $95 \%$ of our target objects. This results in a volume correction factor of $(30 \mathrm{pc} / 24 \mathrm{pc})^{3}=2$ and a Malmquist-corrected binary fraction of $5 /(36 \times 2)$ or $7_{-2}^{+4} \%$.

The possibility that there were faint companions, both stellar and non-stellar, not detected due to instrument insensitivity is a real one. The curve in Figure 7 shows the instrumental sensitivity of our sample in the speckle noise limited region $\left(<1^{\prime \prime}\right.$, $30 \mathrm{AU}$ for a star assumed $30 \mathrm{pc}$ away) as a function of mass ratio and projected separation in AU. It is based on modelling of a 5 Gyr (typical of the ages expected for field stars Gizis et al. (2000)), M6.5 dwarf placed at $30 \mathrm{pc}$ (typical of the distances of our discovered binaries) observed at the 8-m Gemini North telescope. We use the DUSTY models to convert $\Delta H$ magnitudes to mass ratios. The reason we convert to mass ratios is because it allows us to use the observed mass ratio distribution for VLM binaries (Close et al. 2003) to predict the number of missed 
companions with $\mathrm{q} \geq 0.6$. The 5 large asterisks in Figure 7 represent the 5 discovered binary systems from this survey. Interestingly, they are all found in the upper left corner of the sensitivity curve. The fact that some are so near the curve strongly infers that binaries just below the sensitivity curve were most likely missed.

To apply an instrument-sensitivity correction we need to estimate how many binaries went undetected in our sample. We generate a Monte Carlo simulation of 11670 synthetic companions with the binary properties of VLM systems. For our model we use the mass ratio and separation distributions for VLM binaries and assume that the distributions are independent. For the mass ratio distribution we assume a power law decline from unity to 0.6 from Close et al. (2003). We create the separation distribution profile by plotting the 42 most tightly separated and resolved VLM $\left(\mathrm{M}_{t o t}<0.19 \mathrm{M}_{\odot}\right)$ binaries currently known (see Figure 8; Table 4). Originally presented in Close et al. (2003), we update the list of all known VLM binaries and present it in Table 4. The definition of VLM binary having a total mass of $<0.19 \mathrm{M}_{\odot}$ is selected to ensure that the binary components are of spectral type M6.0 or later and hence differentiated from more massive systems. The peak of this distribution, $\sim 5 \mathrm{AU}$, is much tighter than the $\sim 30 \mathrm{AU}$ distribution peak of slightly more massive M0-M4 dwarfs (Fischer \& Marcy 1992) and solar-mass stars (Duquennoy \& Mayor 1991). The separation distribution is bound by the smallest separation the instruments were able to obtain in $\mathrm{H}$ band $\left(\sim 00^{\prime} 08 \times 30 \mathrm{pc}\right)$ on the near side and the empirically sampled wider separation encompassing $95 \%$ of known VLM binaries (Table 4) on the far side.

From this sample of nearly 12,000 simulated companions, $21 \%$ were below the instrument sensitivity curve (but above the instrument sensitivity mass ratio cutoff of $q=0.6$ ) as shown in Figure 7. With 5 detected binaries, this predicts 1.3 companions were missed in our survey. Hence we conclude that the binary fraction for M6.0-M7.5 stars is $(5+1.3) / 36 / 2$ or $9_{-3}^{+4} \%$. It should be pointed out, however, that the true fraction is most certainly larger than this figure since we cannot rule out the possibility of the existence of low q binaries due to the sensitivity of this survey. Therefore, our reported binary fraction, accurate within the uncertainties for M6.0-M7.5 dwarfs for separations $\gtrsim 3 \mathrm{AU}$, represents a low-end estimate to the intrinsic binary fraction.

With slightly improved statistics, this latest result for the binary fraction of M6.0-M7.5 stars is now more comparable with those of later spectral types: $15 \pm 7 \%$ for late M/early L dwarfs (Close et al. 2003), $10-15 \%$ and $15 \pm 5 \%$ for L dwarfs (Bouy et al. 2003; Gizis et al. 2003, respectively) and $9_{-4}^{+15 \%}$ for T dwarfs (Burgasser et al. 2003). These cooler dwarfs including the ones presented here all have binary fractions significantly lower than the $\sim 32 \%$ observed for earlier M0-M4 dwarfs (Fischer \& Marcy 1992) and the $\sim 50 \%$ for solar-mass stars (Duquennoy \& Mayor 1991) over the same a > 3 AU separation range. Our conclusion 
from Paper I is strengthened: for spectral type M6.0-M7.5 binary systems with separations $3 A U<a<300 \mathrm{AU}$ the binary fraction from our survey is $9_{-3}^{+4} \%$, statistically consistent with cooler $M, L$, and $T$ stars and significantly less common than that of $G$ and early $M$ stars.

\subsection{The Separation Distribution Function}

The 2M 0429 and 2M 1847 binary systems have best-estimate projected separations of $6 \mathrm{AU}$ and $2 \mathrm{AU}$, respectively. In our total M6.0-M7.5 sample, we detect no binary separations wider than $10 \mathrm{AU}$ (Table 3). When we analyze the semimajor axis separations of these binaries we observe that there are no projected VLM binary separations $>15 \mathrm{AU}$. With our survey sensitive out to $\sim 15^{\prime \prime}$ from the central star, this result appears to be real and not a result of a sensitivity selection effect. When examining the entire VLM sample of known binary systems from the literature (44, Table 4), only three objects are currently known to have a projected separation $>15 \mathrm{AU}$. This indicates that while wide VLM binaries of $\mathrm{q}>0.6$ can exist, they are rare.

The median projected separation of our entire binary sample is $\sim 5 \mathrm{AU}$, consistent with the $\sim 4$ AU peak distribution of late M/early L binaries (Close et al. 2003), L dwarfs (Gizis et al. 2003; Bouy et al. 2003), and T dwarfs (Burgasser et al. 2003). This contrasts significantly with the $\sim 30$ AU broad separation peak of early M and G dwarfs (Fischer \& Marcy 1992; Duquennoy \& Mayor 1991). We conclude that the projected semimajor axes of M6.0-M7.5 binaries appear consistent with those of late $M, L$, and $T$ dwarf systems but are significantly smaller on average than early $M$ and $G$ stars.

\section{Summary}

We have conducted the largest flux limited $\left(\mathrm{K}_{s}<12 \mathrm{mag}\right)$ survey of nearby M6.0-M7.5 dwarfs using the Keck II, Gemini North, Subaru, and VLT AO systems. In this paper we present our 2 latest binary discoveries, 2M 0479 and 2M 1847, observed at the VLT and Subaru facilities, respectively. When added to our initial results from Paper I, the overall survey consists of 36 stars with 5 discovered binary systems. The 2 new components are of relatively equal mass $(\mathrm{q}>0.8)$ with average projected separations of 2 and $6 \mathrm{AU}$. While none of the binaries have yet been confirmed by common proper motions, they are almost certainly bound based on space density arguments of very red companions. We have used observational and statistical arguments to characterize the VLM binary fraction and separations that contribute additional empirical constraints to binary formation mechanisms: 
- We estimate the binary frequency of spectral type M6.0-M7.5 main sequence stars for separations a $>3 \mathrm{AU}$ from this survey to be $9_{-3}^{+4} \%$. The figure is statistically consistent with later type ultracool M, L, and T dwarfs. The frequency is significantly less than that measured in studies of earlier $M$ and $G$ dwarfs, inferring that the binary fraction of stars is a function of the spectral type of the central star.

- The separations of the 5 binary systems from our sample are all $<10$ AU. Projected separations of known VLM binaries $>15$ AU are rare. This survey's median separation of $5 \mathrm{AU}$ is consistent with the separations of later type M, L, and T dwarfs (separation peak $\sim 4 \mathrm{AU})$. This is in stark contrast with the broad peak separations of $\sim 30 \mathrm{AU}$ for the more massive $\mathrm{M}$ and $\mathrm{G}$ binaries.

We thank Adam Burgasser for elucidating discussion regarding binary fraction statistics, Chien Peng for suggestions regarding Monte Carlo simulations, and Dan Potter for IDL assistance. K.L.C. acknowledges support from a NSF Graduate Research Fellowship. This

publication makes use of data products from the Two-Micron All-Sky Survey, which is a joint project of the University of Massachusetts and the Infrared Processing and Analysis Center/California Institute of Technology, funded by NASA and the NSF.

\section{REFERENCES}

Bate, M. R., Bonnell, I. A., \& Bromm, V. 2002, MNRAS, 332, L65

Basri, G. \& Martín, E. 1999, AJ, 118, 2460

Bouy, H., Brandner W., Martín, E., Delfosse, X., Allard, F., \& Basri, G. 2003, AJ, in press

Bouy, H. et al. 2004, A\&A, in pres

Burgasser, A. J., Kirkpatrick, J. D., Reid, I. N., Brown, M. E., Miskey, C. L., \& Gizis, J. E. 2003, AJ, 586, 512

Burrows, A., Hubbard, W. B., Lunine, J. I., \& Liebert, J. 2001, Reviews of Modern Physics, 73,3

Burrows, A., Sudarsky, D, \& Lunine, J. I. 2003, ApJ, 596, 587

Caloi, V., Cardini, D., D’Antona, F., Badiali, M., Emanuele, A., \& Mazzitelli, I. 1999, A\&A, 351,925 
Chabrier, G., Baraffe, I., Allard, F., \& Hauschildt, P. 2000, ApJ, 542, 464

Chauvin, G. et al. 2004, A\&A, accepted

Close, L. M., Potter, D., Brandner, W., Lloyd-Hart, M., Liebert, J., Burrows, A., \& Siegler, N. 2002a, ApJ, 566, 1095

Close, L. M., Siegler, N., Potter, D., Brandner, W., \& Liebert, J. 2002b, ApJ, 567, L53

Close, L. M., Siegler, N., Freed, M., \& Biller, B. 2003, ApJ, 587, 407

Cruz, K. L., Reid, I. N., Liebert, J., Kirkpatrick, J. D., \& Lowrance, P. J. 2003, AJ, 126, 2421

Dahn et al. 2002, AJ, 124, 1170

Delfosse et al. 1997,A\&A, 327, L25

Duquennoy, A. \& Mayor, M. 1991, A\&A, 248, 485

Fischer, D.A. \& Marcy, G. W. 1992, ApJ, 396, 178

Freed, M., Close, L., \& Siegler, N. 2003, ApJ, 584, 453

Gizis, J.E., Monet, D.G., Reid, I.N., Kirkpatrick, J.D., Liebert, J., \& Williams, R.J. 2000, AJ, 120, 1085

Gizis, J.E. et al. 2003, AJ, 125, 3302

Graves, J.E., Northcott, M.J., Roddier, F.J., Roddier, C.A., Close, L.M. 1988, Proc. SPIE Vol. 3353, p. 34-43. Adaptive Optical System Technologies, D. Bonaccini, R.K. Tyson, Eds.

Guinan, E. F. \& Ribas, I. 2001, ApJ, 546, L43

Hawley, S., Reid, I. N., Gizis, J. E., \& Byrne, P. B. 1999, Solar and Stellar Activity: Similarities and Differences, ASP Conference Series 158, ed. C. J. Butler \& J. G. Doyle. ISBN 1-886733-78-3, p.63

Jones, B. F., Fischer, D. A., \& Stauffer, J. R. 1996, AJ, 112, 1562

Kenworthy, M., Hofmann, K-H., Close, L., Hinz, P., Mamajek, E., Schertl, D., Weigelt, G., Angel, R., Balega, Y.Y., Hinz, J., Rieke, G. 2001, ApJ, 554, L67

Kirkpatrick, J. D., Henry, T. J., \& McCarthy, D. W. 1991, ApJS, 77, 417 
Kirkpatrick et al. 1999, ApJ, 519, 802

Kirkpatrick, J. D., Reid, I. N., Liebert, J., Gizis, J. E., Burgasser, A. J., Monet, D. G., Dahn, C. C., Nelson, B., Williams, R. J. 2000, AJ, 120, 447

Koerner, D. W., Kirkpatrick, J. Davy, McElwain, M. W., Bonaventura, N. R. 1999, ApJ, 526, L25

Lane, B.F., Zapatero Osorio, M.R., Britton, M.C., Martin, E.L., Kulkarni, S.R. 2001, ApJ, $560,390 \mathrm{~L}$

Leinert, Ch., Jahreiss, H., Woitas, J., Zucker, S., Mazeh, T., Eckart, A., \& Kohler, R. 2001, A\&A, 367, 183

Luhman, K. L. 2004, ApJ, in press

Martín, E. L., Brandner, W., \& Basri, G. 1999, Science, 283, 1718

Martín, E. L. et al. 1998, ApJ, 543, 299

Martín, E. L., Navascués, D. B. y, Baraffe, I., Bouy, H., \& Dahm, S. 2003, ApJ, 594, 525

McCaughrean, M. J., et al. 2003, A\&A, 413, 1029

Potter et al. 2002, ApJ, 567, L133

Pravdo, S. H., et al. 1999, AJ, 117, 1616

Reid, I. N. 1993, MNRAS, 265, 785

Reid, I. N. \& Gizis, J. E. 1997a, AJ, 113, 2246

Reid, I. N. \& Gizis, J. E. 1997b, AJ, 114, 1992

Reid, I. N. \& Hawley, S. L. 1999, AJ, 117, 343

Reid, I. N. \& Mahoney, S. 2000, MNRAS, 316, 827

Reid, I. N., Gizis, J. E., Kirkpatrick, J. D., \& Koerner, D. W. 2001, AJ, 121, 489

Reid, I. N., Kirkpatrick, J. D., Liebert, J., Gizis, J. E., Dahn, C. C., \& Monet, D. G. 2002, AJ, 124,519

Reid, I. N., et al. 2003, AJ, 125, 354

Reipurth, B. \& Clarke, C. 2001, AJ, 122, 432 
Roddier, F. 1999, Adaptive Optics in Astronomy, Cambridge University Press

Siegler, N., Close, L. M., \& Freed M. 2002, Proc. SPIE Vol. 4839, 114, Adaptive Optics Systems Technologies II, P. Wizinowich, D. Bonaccini, Eds.

Siegler, N., Close, L. M., Mamajek, E. E., \& Freed, M. 2003, ApJ, 598, 1265 (Paper I)

Simon, M., Close, L. M., \& Beck, T. 1999, AJ, 117, 1375

Tamura., et al. 2000, Proc. SPIE Vol. 4008, 1153, Optical and IR Telescope Instrumentation and Detectors, Iye, M. \& Moorwood, A. F., Eds

Teegarden, B. J., et al. 2003, ApJ. 589, L51

White, R. J., \& Ghez, A. M. 2001, ApJ, 556, 265

Wielen, R. 1974, Highlight Astron., 3, 375

Wizinowich, P., et al. 2000, PASP, 112, 315 
Table 1. M6.0-M7.5 Stars Observed with No Likely Physical Companion Detections Between 0.1" $-15^{\prime \prime a}$

\begin{tabular}{|c|c|c|c|c|}
\hline 2MASS Name & Other Name & $\mathrm{K}_{s}$ & Spectral Type & Reference \\
\hline $\mathrm{J} 0253008+165253^{\mathrm{b}}$ & SO $025300.5+165258$ & 7.59 & M6.5 & 5 \\
\hline 2MASSI J0330050+240528 & LP 356-770 & 11.36 & M7.0 & 1 \\
\hline 2MASSI J0435161-160657 & LP775-31 & 9.34 & M7.0 & 2 \\
\hline 2MASSI J0752239+161215 & & 9.82 & M7.0 & 2 \\
\hline 2MASSI J0818580+233352 & & 11.13 & M7.0 & 1 \\
\hline 2MASSW J0952219-192431 & & 10.85 & M7.0 & 1 \\
\hline 2MASSW J1016347+275150 & LHS 2243 & 10.95 & M7.5 & 1 \\
\hline 2MASSI J1024099+181553 & & 11.21 & M7.0 & 1 \\
\hline 2MASSW J1049414+253852 & & 11.39 & M6.0 & 1 \\
\hline 2MASSI J1124532+132253 & & 10.03 & M6.5 & 2 \\
\hline 2MASSW J1200329+204851 & & 11.82 & M7.0 & 1 \\
\hline 2MASSW J1237270-211748 & & 11.64 & M6.0 & 1 \\
\hline 2MASSW J1246517+314811 & LHS 2632 & 11.23 & M6.5 & 1 \\
\hline 2MASSI J1253124+403404 & & 11.20 & M7.5 & 4 \\
\hline 2MASSW J1336504+475131 & & 11.63 & M7.0 & 1 \\
\hline 2MASSW J1344582+771551 & & 11.83 & M7.0 & 1 \\
\hline 2MASSI J1356414+434258 & & 10.63 & M7.5 & 2 \\
\hline 2MASSI J1431304+171758 & & 11.16 & M6.5 & 2 \\
\hline 2 MASSI J1521010+505323 & & 10.92 & M7.5 & 2 \\
\hline 2MASSP J1524248+292535 & & 10.15 & M7.5 & 3 \\
\hline 2MASSW J1527194+413047 & & 11.47 & M7.5 & 3 \\
\hline 2MASSW J1543581+320642 & LP $328-36$ & 11.73 & M7.5 & 1 \\
\hline 2MASSW J1546054+374946 & & 11.42 & M7.5 & 1 \\
\hline 2MASSW J1550381+304103 & & 11.92 & M7.5 & 1 \\
\hline 2MASSW J1757154+704201 & LP 44-162 & 10.37 & M7.5 & 1 \\
\hline 2MASSW J2052086-231809 & LP $872-22$ & 11.26 & M6.5 & 1 \\
\hline 2MASSW J2221544+272907 & & 11.52 & M6.0 & 1 \\
\hline 2MASSW J2233478+354747 & LP 288-31 & 11.88 & M6.0 & 1 \\
\hline 2MASSI J2235490+184029 & LP $460-44$ & 11.33 & M7.0 & 1 \\
\hline 2MASSW J2306292-050227 & & 10.29 & M7.5 & 1 \\
\hline 2MASSW J2313472+211729 & LP $461-11$ & 10.42 & M6.0 & 1 \\
\hline
\end{tabular}

${ }^{\text {a}}$ For near-equal mass companions. For smaller companion masses with $\mathrm{q}<0.8$, sensitivity is a function of distance. See Figures 3 and 7 .

${ }^{\mathrm{b}}$ Results from this paper; otherwise, Paper I.

References. - (1) Gizis et al. (2000) (2) Cruz et al. (2003) (3) Reid et al. (2002) (4) Kirkpatrick, Henry, \& McCarthy (1991) (5) Teegarden et al. (2003). 
Table 2. The New Binary Systems

\begin{tabular}{|c|c|c|c|c|c|c|c|}
\hline System & $\Delta J$ & $\Delta H$ & $\Delta K_{s}$ & Sep. (mas) & P.A. (deg) & Date Observed (UT) & Telescope \\
\hline $\mathrm{LP} 415-20^{\mathrm{a}}$ & $0.84 \pm 0.15$ & $0.77 \pm 0.10$ & $0.66 \pm 0.06$ & $119 \pm 8$ & $91.2 \pm 0.7$ & 2002 Feb. 07 & Gemini North \\
\hline LP $475-855^{\mathrm{b}}$ & $0.48 \pm 0.05$ & $0.43 \pm 0.04$ & $0.48 \pm 0.03$ & $294 \pm 5$ & $131.6 \pm 0.5$ & 2001 Sep. 22 & Gemini North \\
\hline 2MASSI J0429184-312356 ${ }^{\mathrm{c}}$ & $1.20 \pm 0.12$ & $1.10 \pm 0.08$ & $0.98 \pm 0.08$ & $531 \pm 2$ & $298.9 \pm 0.2$ & 2003 Feb. 13 & VLT \\
\hline 2MASSW J1750129+442404 & $0.74 \pm 0.15$ & $0.73 \pm 0.15$ & $0.64 \pm 0.10$ & $158 \pm 5$ & $339.6 \pm 0.7$ & 2002 Apr. 25 & Gemini North \\
\hline 2 MASSI J1847034+552243 & $0.26 \pm 0.18$ & $0.34 \pm 0.15$ & $0.16 \pm 0.10$ & $82 \pm 5$ & $91.1 \pm 1.4$ & 2003 July 10 & Subaru \\
\hline
\end{tabular}

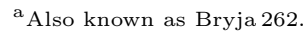

${ }^{\mathrm{b}}$ Also known as [LHD94] 042614.2+13312 and 2MASSW J0429028+133759.

${ }^{\mathrm{c}}$ Results from this paper; otherwise, Paper I. 
Table 3. Summary of the Binaries' A and B Components

\begin{tabular}{|c|c|c|c|c|c|c|c|c|c|}
\hline Name & $J$ & $H$ & $K_{s}$ & $M_{K_{s}}{ }^{\mathrm{a}}$ & $\mathrm{SpT}^{\mathrm{b}}$ & $\mathrm{d}_{\text {phot }}(\mathrm{pc})^{\mathrm{c}}$ & Mass $\left(\mathrm{M}_{\odot}\right)^{\mathrm{d}}$ & Sep. $(A U)^{e}$ & $P(y r)^{f}$ \\
\hline LP $415-20 \mathrm{~A}$ & $13.09 \pm 0.06$ & $12.47 \pm 0.05$ & $12.12 \pm 0.04$ & $9.72 \pm 0.38$ & M7.0 & \multirow[t]{2}{*}{$30 \pm 5$} & $0.097_{-0}^{+0}$ & \multirow[t]{2}{*}{$3.6 \pm 0.7$} & \multirow[t]{2}{*}{$23_{-6}^{+7}$} \\
\hline LP 415-20B & $13.93 \pm 0.16$ & $13.24 \pm 0.11$ & $12.78 \pm 0.08$ & $10.37 \pm 0.39$ & M9.5 & & $0.081_{-0.010}^{+0.009}$ & & \\
\hline LP $475-855 \mathrm{~A}$ & $13.21 \pm 0.04$ & $12.54 \pm 0.04$ & $12.18 \pm 0.04$ & $9.84 \pm 0.36$ & M7.5 & \multirow[t]{2}{*}{$29 \pm 5$} & $0.093_{-0.0}^{+0.0}$ & \multirow[t]{2}{*}{$8.6 \pm 1.5$} & \multirow[t]{2}{*}{$86_{-19}^{+20}$} \\
\hline LP $475-855 B$ & $13.69 \pm 0.07$ & $12.97 \pm 0.06$ & $12.66 \pm 0.05$ & $10.32 \pm 0.36$ & M9.5 & & $0.082_{-0.009}^{+0.009}$ & & \\
\hline $2 \mathrm{M} 0429 \mathrm{~A}^{\mathrm{g}}$ & $11.18 \pm 0.04$ & $10.55 \pm 0.03$ & $10.14 \pm 0.03$ & $9.88 \pm 0.35$ & M7.5 & \multirow[t]{2}{*}{$11 \pm 2$} & $0.094_{-0.011}^{+0.009}$ & \multirow[t]{2}{*}{$6.0 \pm 1.0$} & \multirow[t]{2}{*}{$50_{-11}^{+12}$} \\
\hline $2 \mathrm{M} \mathrm{0429 \textrm {B } ^ { \mathrm { g } }}$ & $12.38 \pm 0.13$ & $11.65 \pm 0.09$ & $11.12 \pm 0.07$ & $10.86 \pm 0.36$ & L1.0 & & $0.079_{-0.018}^{+0.005}$ & & \\
\hline $2 \mathrm{M} 1750 \mathrm{~A}$ & $13.23 \pm 0.06$ & $12.62 \pm 0.06$ & $12.24 \pm 0.05$ & $9.77 \pm 0.39$ & M7.5 & \multirow[t]{2}{*}{$31 \pm 6$} & $0.097_{-0.012}^{+0.018}$ & \multirow[t]{2}{*}{$4.9 \pm 0.9$} & \multirow[t]{2}{*}{$36_{-9}^{+10}$} \\
\hline $2 \mathrm{M} 1750 \mathrm{~B}$ & $13.97 \pm 0.16$ & $13.35 \pm 0.16$ & $12.88 \pm 0.11$ & $10.41 \pm 0.41$ & M9.5 & & $0.085^{+}$ & & \\
\hline $2 \mathrm{M} 1847 \mathrm{~A}^{\mathrm{g}}$ & $12.55 \pm 0.08$ & $11.87 \pm 0.07$ & $11.58 \pm 0.05$ & $9.72 \pm 0.41$ & M7.0 & \multirow[t]{2}{*}{$23 \pm 4$} & $0.098_{-0.012}^{+0.022}$ & \multirow{2}{*}{$1.9 \pm 0.4$} & \multirow[t]{2}{*}{$9_{-2}^{+3}$} \\
\hline $2 \mathrm{M} 1847 \mathrm{~B}^{\mathrm{g}}$ & $12.81 \pm 0.20$ & $12.21 \pm 0.16$ & $11.74 \pm 0.11$ & $9.88 \pm 0.42$ & M7.5 & & $0.094_{-0.013}^{+0.014}$ & & \\
\hline
\end{tabular}

${ }^{\mathrm{a}} \mathrm{M}_{K_{s}}=7.65+2.13\left(\mathrm{~J}-K_{s}\right)$ with a rms $\sigma_{M_{K s}}=0.33$ derived in Paper I (§4.2); relationship is valid for M6.5 $<\mathrm{SpT}<\mathrm{L} 1$.

${ }^{\mathrm{b}}$ Spectral type estimated by $\mathrm{SpT}=3.54 \mathrm{M}_{K_{S}}-27.20$ with \pm 1.5 spectral subclasses of error in these estimates as derived in paper $\mathrm{I}(\S 4.5)$. SpT $=$ 10 is defined as an L0; valid for M6.5 $<\mathrm{SpT}<\mathrm{L} 1$.

${ }^{\mathrm{c}}$ Distances based on $\mathrm{M}_{K_{S}}$ as described in $\S 4.2$.

${ }^{d}$ Mass determination uses the models of Chabrier et al. (2000).

${ }^{\mathrm{e}}$ Projected separations.

${ }^{\mathrm{f}}$ Periods include a 1.26 multiplication of the projected separations compensating for random inclinations and eccentricities (Fischer \& Marcy 1992).

$\mathrm{g}_{\text {This paper; otherwise, Paper I. }}$ 
Table 4. All Known Resolved VLM Binaries ${ }^{\mathrm{a}}$

\begin{tabular}{|c|c|c|c|c|c|c|}
\hline Name & $\begin{array}{l}\text { Sep. }{ }^{b} \\
\text { AU }\end{array}$ & $\begin{array}{c}\text { Est. } \\
S p T_{A} / S p T_{B}\end{array}$ & $\begin{array}{c}\text { Est. } M_{A} \\
M_{\odot}\end{array}$ & $\begin{array}{c}\text { Est. } M_{B} \\
M_{\odot}\end{array}$ & $\begin{array}{c}\text { Est. Period } \\
\text { yr }\end{array}$ & Ref. $^{\mathrm{i}}$ \\
\hline PPL $15^{\mathrm{d}}$ & 0.03 & $\mathrm{M} 7 / \mathrm{M} 8$ & 0.07 & 0.06 & 5.8 days & 1 \\
\hline $\mathrm{Gl} 569 \mathrm{~B}^{\mathrm{e}}$ & 1.0 & M8.5/M9.0 & 0.063 & 0.06 & 3 & 2,3 \\
\hline SDSS 2335583-001304 & $1.1 ?$ & $\mathrm{~L} 1 ? / \mathrm{L} 4 ?$ & 0.079 & 0.074 & 3 & 4 \\
\hline 2MASSI J1112256+354813 & 1.5 & $\mathrm{~L} 4 / \mathrm{L} 6$ & 0.073 & 0.070 & 5 & 4 \\
\hline 2MASSI J1534498-295227 & 1.8 & $\mathrm{~T} 5.5 / \mathrm{T} 5.5$ & 0.05 & 0.05 & 8 & 5 \\
\hline 2MASSI J1847034+552243 & 1.9 & $\mathrm{M} 7 / \mathrm{M} 7.5$ & 0.098 & 0.094 & 9 & This paper \\
\hline 2MASSW J0856479+223518 & 2.0 & $\mathrm{~L} 5 ? / \mathrm{L} 8 ?$ & 0.071 & 0.064 & 8 & 4 \\
\hline DENIS-P J185950.9-370632 & 2.0 & L0/L3 & 0.084 & 0.076 & 7 & 4 \\
\hline HD $130948 B^{d}$ & 2.4 & $\mathrm{~L} 2 / \mathrm{L} 2$ & 0.07 & 0.06 & 10 & 6 \\
\hline$\epsilon$ Indi B & 2.6 & $\mathrm{~T} 1 / \mathrm{T} 6$ & 0.042 & 0.027 & 16 & 16 \\
\hline 2MASSW J0746425+200032 & 2.7 & L0/L1.5 & 0.085 & 0.066 & 11 & $7,17,20$ \\
\hline 2MASSW J1047127+402644 & 2.7 & M8/L0 & 0.092 & 0.084 & 11 & 8,17 \\
\hline DENIS-P J035726.9-441730 & 2.8 & $\mathrm{~L} 2 / \mathrm{L} 4$ & 0.078 & 0.074 & 12 & 4,13 \\
\hline 2MASSW J12255432-2739466 & 3.2 & $\mathrm{~T} 6 / \mathrm{T} 8$ & 0.033 & 0.024 & 23 & 5 \\
\hline 2MASSW J0920122+351742 & 3.2 & $\mathrm{~L} 6.5 / \mathrm{L} 7$ & 0.068 & 0.068 & 16 & 7 \\
\hline LHS $1070 B^{f}$ & 3.4 & M8.5/M9.0 & 0.070 & 0.068 & 16 & 18 \\
\hline LP $415-20$ & 3.5 & M7/M9.5 & 0.095 & 0.079 & 15 & 9, Paper I \\
\hline 2MASSW J1728114+394859 & 3.7 & $\mathrm{~L} 7 / \mathrm{L} 8$ & 0.069 & 0.066 & 19 & 4,13 \\
\hline LHS 2397a & 3.9 & M8/L7.5 & 0.090 & 0.068 & 22 & 10,17 \\
\hline 2MASSW J1426316+155701 & 3.9 & $\mathrm{M} 8.5 / \mathrm{L} 1$ & 0.088 & 0.076 & 19 & 17 \\
\hline 2MASSW J2140293+162518 & 3.9 & $\mathrm{M} 9 / \mathrm{L} 2$ & 0.092 & 0.078 & 22 & 17 \\
\hline 2MASSW J2206228-204705 & 4.4 & M8/M8 & 0.092 & 0.092 & 22 & 17 \\
\hline 2MASSs J0850359+105716 & 4.4 & $\mathrm{~L} 6 / \mathrm{L} 8$ & 0.05 & 0.04 & 30 & 7 \\
\hline 2MASSW J1750129+442404 & 4.8 & $\mathrm{M} 7.5 / \mathrm{L} 0$ & 0.095 & 0.084 & 25 & 9, Paper I \\
\hline DENIS-P J1228.2-1547 & 4.9 & $\mathrm{~L} 5 / \mathrm{L} 5$ & 0.05 & 0.05 & 34 & 11 \\
\hline 2MASSW J1600054+170832 & 5.0 & $\mathrm{~L} 1 / \mathrm{L} 3$ & 0.078 & 0.075 & 29 & 4,13 \\
\hline 2MASSW J1239272+551537 & 5.1 & $\mathrm{~L} 5 / \mathrm{L} 5$ & 0.071 & 0.071 & 31 & 4,13 \\
\hline 2MASSI J0429184-312356 & 6.0 & $\mathrm{M} 7.5 / \mathrm{L} 1$ & 0.094 & 0.079 & 50 & This paper \\
\hline IPMBD 29 & 7.2 & $\mathrm{~L} 1 / \mathrm{L} 4^{\mathrm{g}}$ & 0.045 & 0.038 & 68 & 14 \\
\hline 2MASSW J1146345+223053 & 7.6 & $\mathrm{~L} 3 / \mathrm{L} 4$ & 0.055 & 0.055 & 63 & 12 \\
\hline 2MASSW J1311391+803222 & 7.7 & M8.5/M9 & 0.089 & 0.087 & 51 & 17 \\
\hline CFHT-Pl-12 & 7.8 & $\mathrm{M} 8 / \mathrm{L} 4^{\mathrm{g}}$ & 0.054 & 0.038 & 76 & 14 \\
\hline 2MASSW J1127534+741107 & 8.3 & M8/M9 & 0.092 & 0.087 & 57 & 17 \\
\hline LP $475-855$ & 8.3 & M7.5/M9.5 & 0.091 & 0.080 & 58 & 9, Paper I \\
\hline DENIS-P J0205.4-1159 & 9.2 & $\mathrm{~L} 7 / \mathrm{L} 7$ & 0.07 & 0.07 & 75 & 15 \\
\hline 2MASSW J2101349+175611 & 9.6 & $\mathrm{~L} 7 / \mathrm{L} 8$ & 0.068 & 0.065 & 82 & 4,13 \\
\hline 2MASSW J2147436+143131 & 10.4 & $\mathrm{~L} 0 / \mathrm{L} 2$ & 0.084 & 0.078 & 83 & 4,13 \\
\hline 2MASSW J1449378+235537 & 11.7 & $\mathrm{~L} 0 / \mathrm{L} 3$ & 0.084 & 0.075 & 100 & 4,13 \\
\hline IPMBD 25 & 11.8 & $\mathrm{M} 7 / \mathrm{L} 4^{\mathrm{g}}$ & 0.063 & 0.039 & 126 & 14 \\
\hline DENIS-P J144137.3-094559 & 13.5 & $\mathrm{~L} 1 / \mathrm{L} 1$ & 0.079 & 0.079 & 124 & 4,13 \\
\hline 2MASSW J2331016-040618 e & 15.0 & $\mathrm{M} 8.5 / \mathrm{L} 7$ & 0.093 & 0.067 & 159 & 17 \\
\hline CFHT-Pl-18 & 34.5 & M8/M8 & 0.09 & 0.09 & 641 & 4,19 \\
\hline 2MASSW J1207334-393254 h & 55 & M8/L5-L9.5 & 0.02 & 0.005 & 2600 & 22 \\
\hline 2MASSW J11011926-7732383 & 240 & $\mathrm{M} 7 / \mathrm{M} 8$ & 0.05 & 0.025 & 13600 & 21 \\
\hline
\end{tabular}

${ }^{a}$ We define VLM binaries as systems whose total mass is $<0.19 M_{\odot}$. Very young evolving systems like GG TauBaBb (White \& Ghez 2001) are not included, nor are overluminous systems that are not resolved into 
binaries.

${ }^{\mathrm{b}}$ Projected separation except for the systems where semimajor axes have been measured.

"This "period" is simply an estimate assuming a face-on circular orbit except for the systems where P has been measured.

dPPL 15 is a spectroscopic binary (Basri \& Martín (1999) and is not resolved).

eThese tight binaries have a widely separated more massive primary.

${ }^{\mathrm{f}}$ Part of a triple system.

${ }^{\mathrm{g}}$ Spectral type estimated from the given masses using Pleiades age, effective temperatures from Burrows et al. (2001), and spectral type-temperature relation for ultracool dwarfs from Dahn et al. (2002).

${ }^{\mathrm{h}}$ Parallaxes and common proper motions have yet to be confirmed

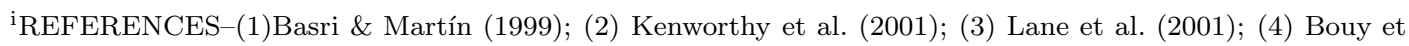
al. (2003); (5) Burrows, Sudarsky, \& Lunine (2003); (6) Potter et al. (2002); (7) Reid et al. (2001); (8) Reid et al. (2002); (9) Siegler et al. (2003); (10) Freed, Close, \& Siegler (2003); (11) Martin et al. (1999); (12) Koerner et al. (1999); (13) Gizis et al. (2003); (14) Martin et al. (2003); (15) Delfosse et al. (1997); (16) McCaughrean et al. (2004); (17) Close et al. (2003); (18) Leinert et al. (2001); (19) Martin et al. (2000); (20) Bouy et al. (2004); (21) Luhman (2004); (22) Chauvin et al. (2004) 


\section{M0420}

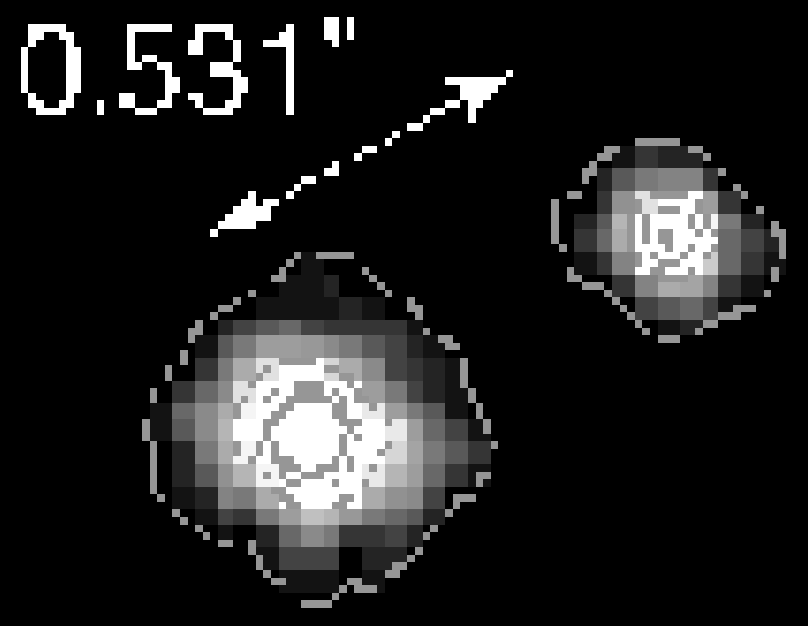

Fig. 1.- An $8 \times 0.5 \mathrm{~s}$ image of the newly discovered binary system $2 \mathrm{M} 0429$ shown in the $\mathrm{K}_{s}$ band; observed on 2003 February 13 (UT) at the VLT. The platescale is $0^{\prime \prime} .0271$ pixel $^{-1}$. The contours are linear at the $80,60,40$, and $20 \%$ levels; north is up and east is to the left. 

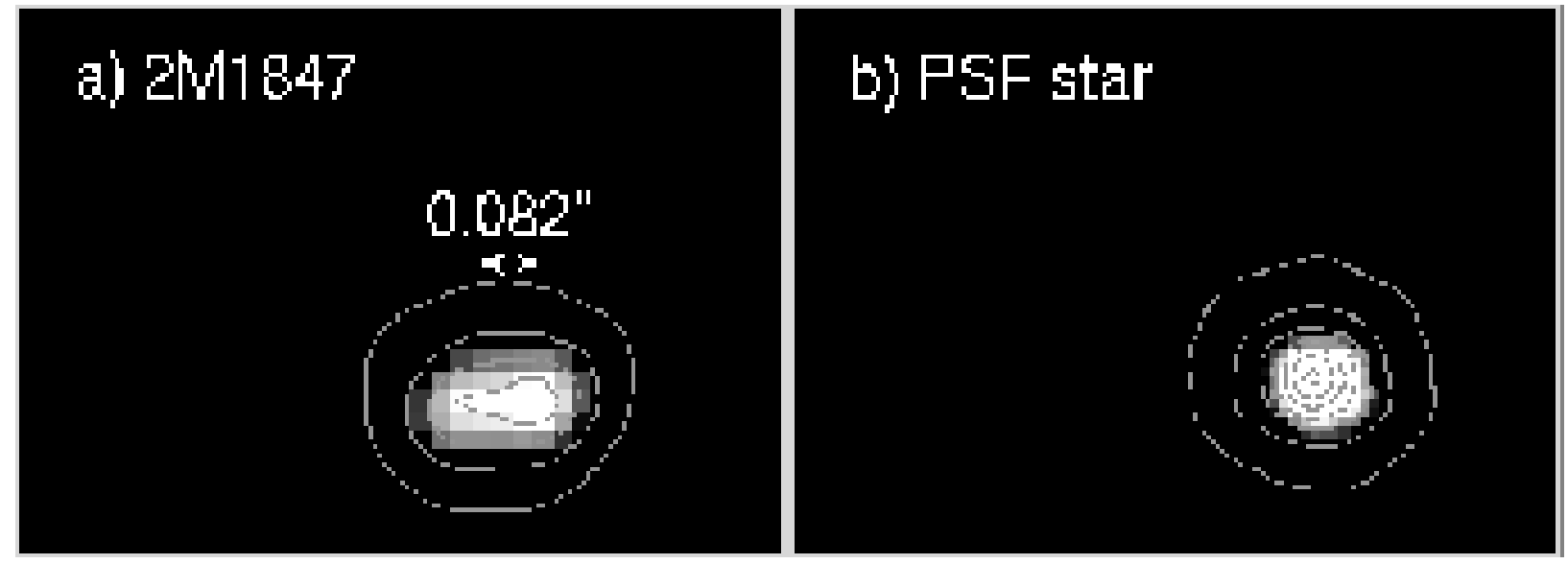

Fig. 2.- (a) A $12 \times 10 \mathrm{~s}$ image of the newly discovered binary system $2 \mathrm{M} 1847$ shown in the $\mathrm{K}_{s}$ band; observed on 2003 July 10 (UT) at Subaru. The platescale is $0^{\prime \prime} .0217 \mathrm{pixel}^{-1}$. The contours are linear at the 80,60, 40, and 20\% levels. (b) For comparison, the PSF star $2 \mathrm{M} 0253$ observed in the same evening and at the same airmass is displayed. The contours are linear at the $85,75,65,55,45,30$, and $15 \%$ levels. In both images, north is up and east is to the left. 


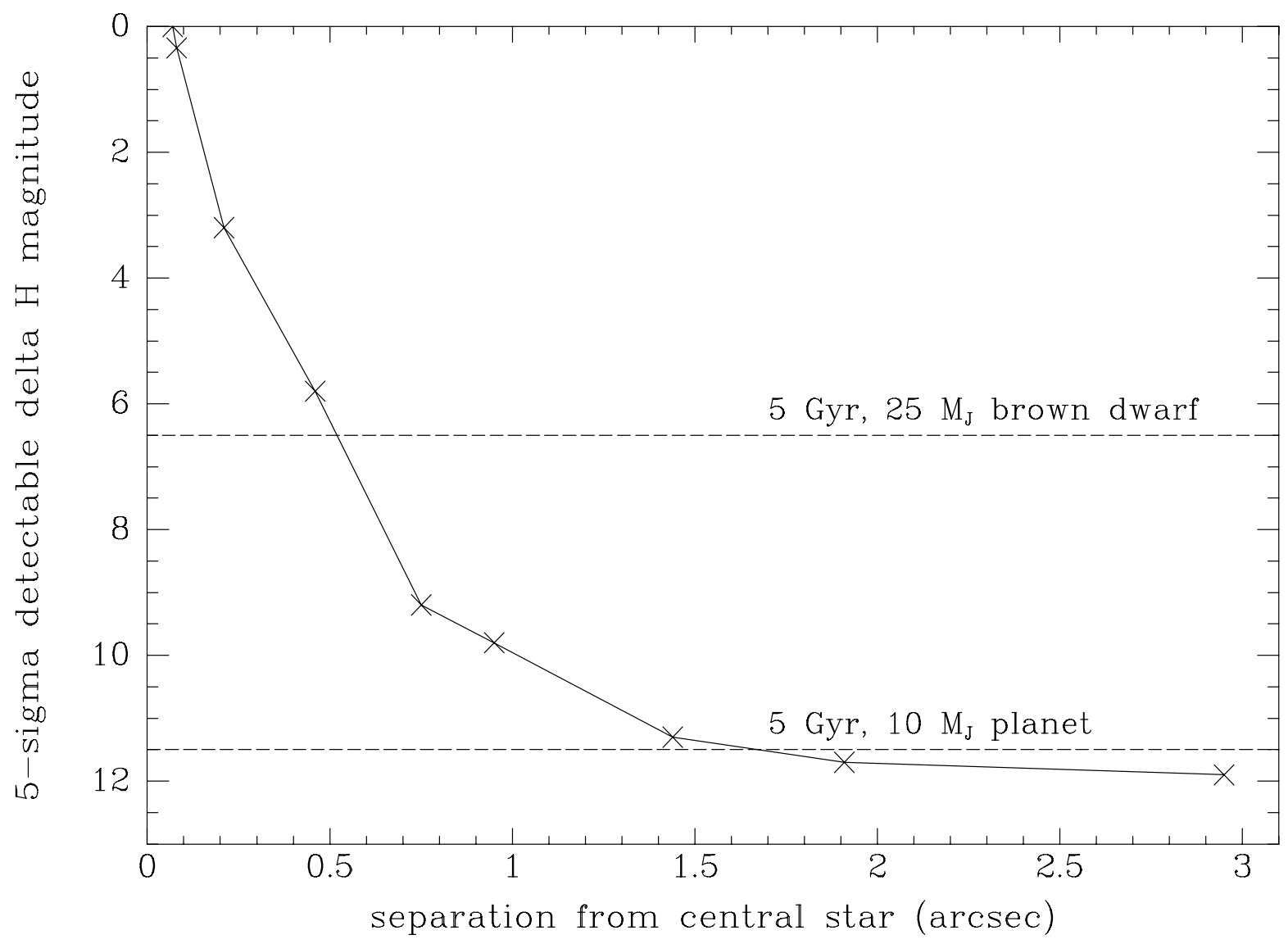

Fig. 3.- Instrument sensitivity curve showing $5 \sigma \Delta \mathrm{H}$ detection versus distance in arcsec from the very nearby M6.5 star 2M 0253 . Total integration time is 24 min using Keck II AO. The "crosses" indicate the $5 \sigma$ sensitivity limits of our data to simulated faint companions. The upper horizontal dashed line corresponds to a $5 \mathrm{Gyr}$ old, $25 \mathrm{M}_{J}$ brown dwarf using the models of Burrows, Sudarsky, \& Lunine (2003) while the lower horizontal dashed line corresponds to a $5 \mathrm{Gyr}$ old, $10 \mathrm{M}_{J}$ planet. We were sensitive to the detection of a $10 \mathrm{M}_{J}$ planet at $4 \mathrm{AU}$ and a $25 \mathrm{M}_{J}$ brown dwarf at $1.5 \mathrm{AU}$ (assuming $2 \mathrm{M} 0253$ is only $3.6 \mathrm{pc}$ away and 5 Gyr old). 


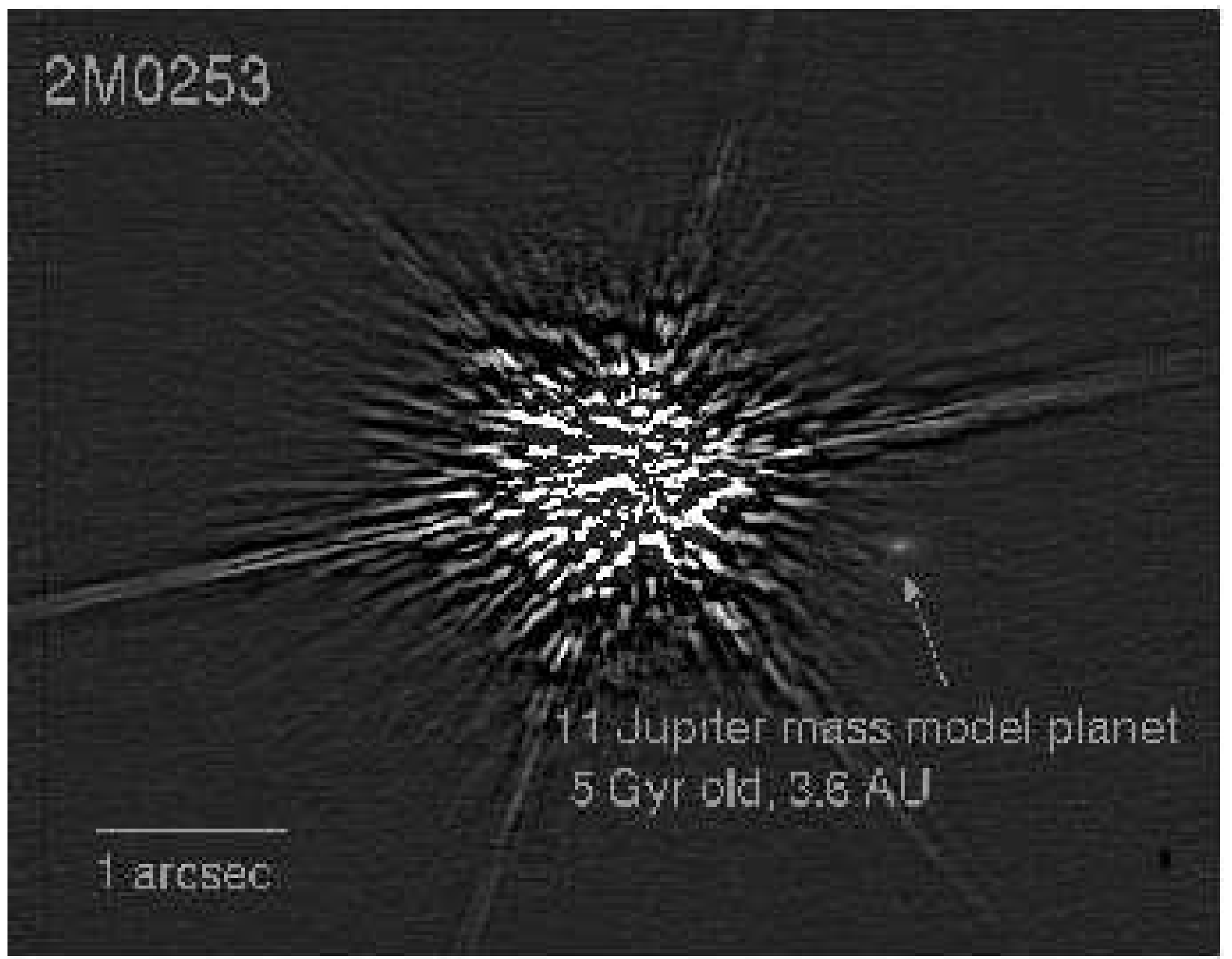

Fig. 4.- Reduced 24 min image of 2M 0253 observed at Keck II on 2003 July 14 (UT) with its low spatial frequencies removed (unsharp masked). The simulated $5 \sigma$ companion is 33,000 times fainter than the central star $(\Delta \mathrm{H}=11.3)$ at only $3.6 \mathrm{AU}\left(1.5^{\prime \prime}\right)$. Teegarden et al. (2003) report $\mathrm{H}=7.9$ for the central star which allows detection to a $5 \mathrm{Gyr}$ old, $11 \mathrm{M}_{J}$ planet according to the models of Burrows, Sudarsky, \& Lunine (2003). Also visible are the residuals from the 6 spider arms and super speckles. To within our sensitivity limits we find no companions to $2 \mathrm{M} 0253$. 


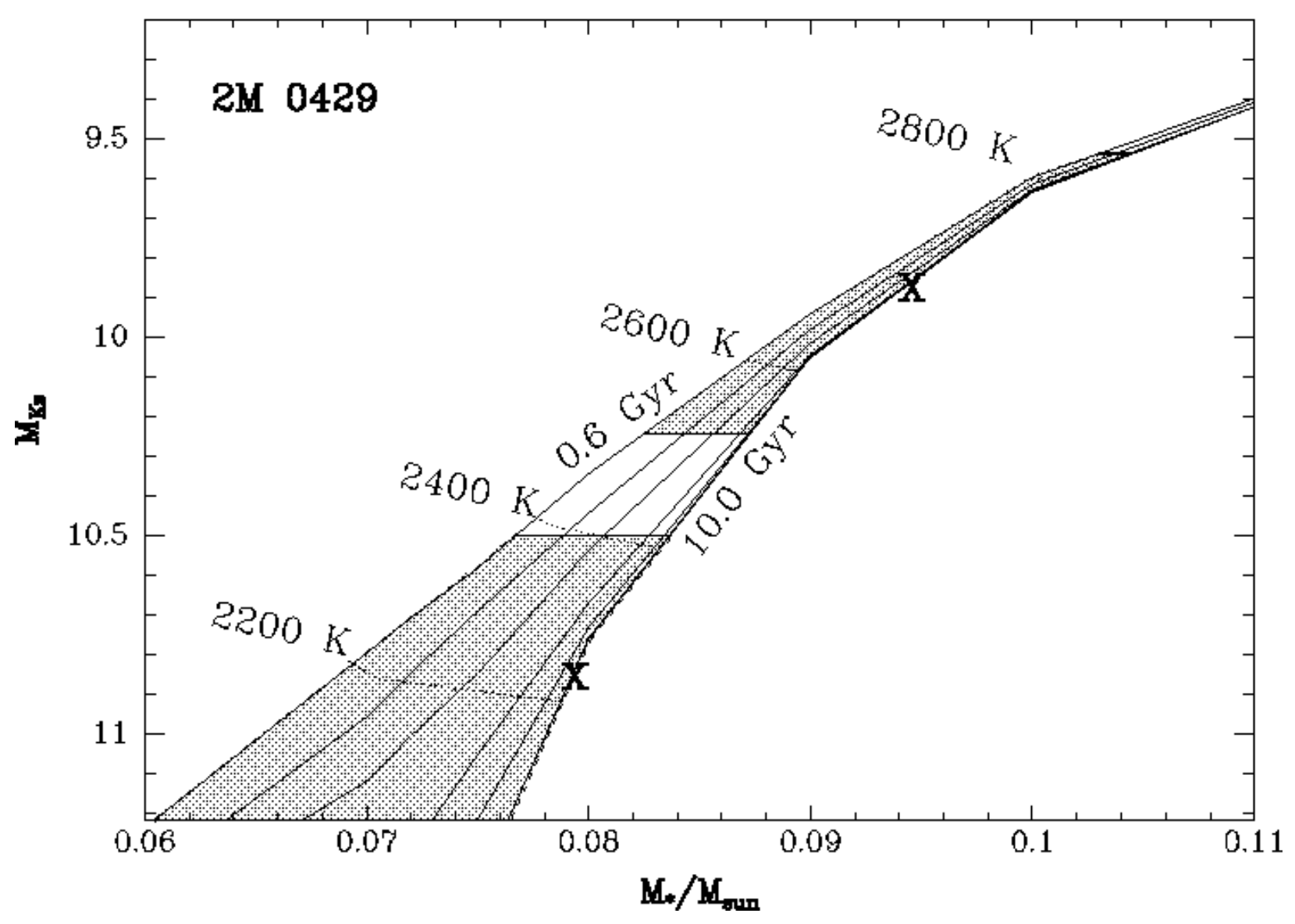

Fig. 5.- Chabrier et al. (2000) DUSTY stellar and substellar evolutionary tracks custom integrated over the $\mathrm{K}_{s}$ bandpass $([\mathrm{m} / \mathrm{H}]=0)$. The best-guess values of the individual binary components of 2M 0429 are indicated by the bold "crosses" with the primary at the top right and the companion lower and to the left. The shaded polygons enclose each components' region of uncertainty. The components' derived $\mathrm{M}_{K_{s}}$ is listed in Table 3. With no knowledge of the binary's age, we conservatively assign a mean age of 5 Gyr and uncertainties spanning the range of ages in the solar neighborhood (0.6-7.5 Gyr; Caloi et al. 1999). The model suggests a primary mass of $0.094_{-0.011}^{+0.010} \mathrm{M}_{\odot}$ and a temperature of $2690_{-170}^{+160} \mathrm{~K}$. For the companion, the model predicts a mass of $0.079_{-0.018}^{+0.005} \mathrm{M}_{\odot}$ and a temperature of $2240_{-260}^{+190} \mathrm{~K}$. The isochrones plotted are (left to right) 0.6, 0.65, 0.7, 0.85, 1.2, 1.7, 3.0, 5.0, 7.5, and $10.0 \mathrm{Gyr}$ (the oldest 4 isochrones are indistinguishable at the given scaling). 


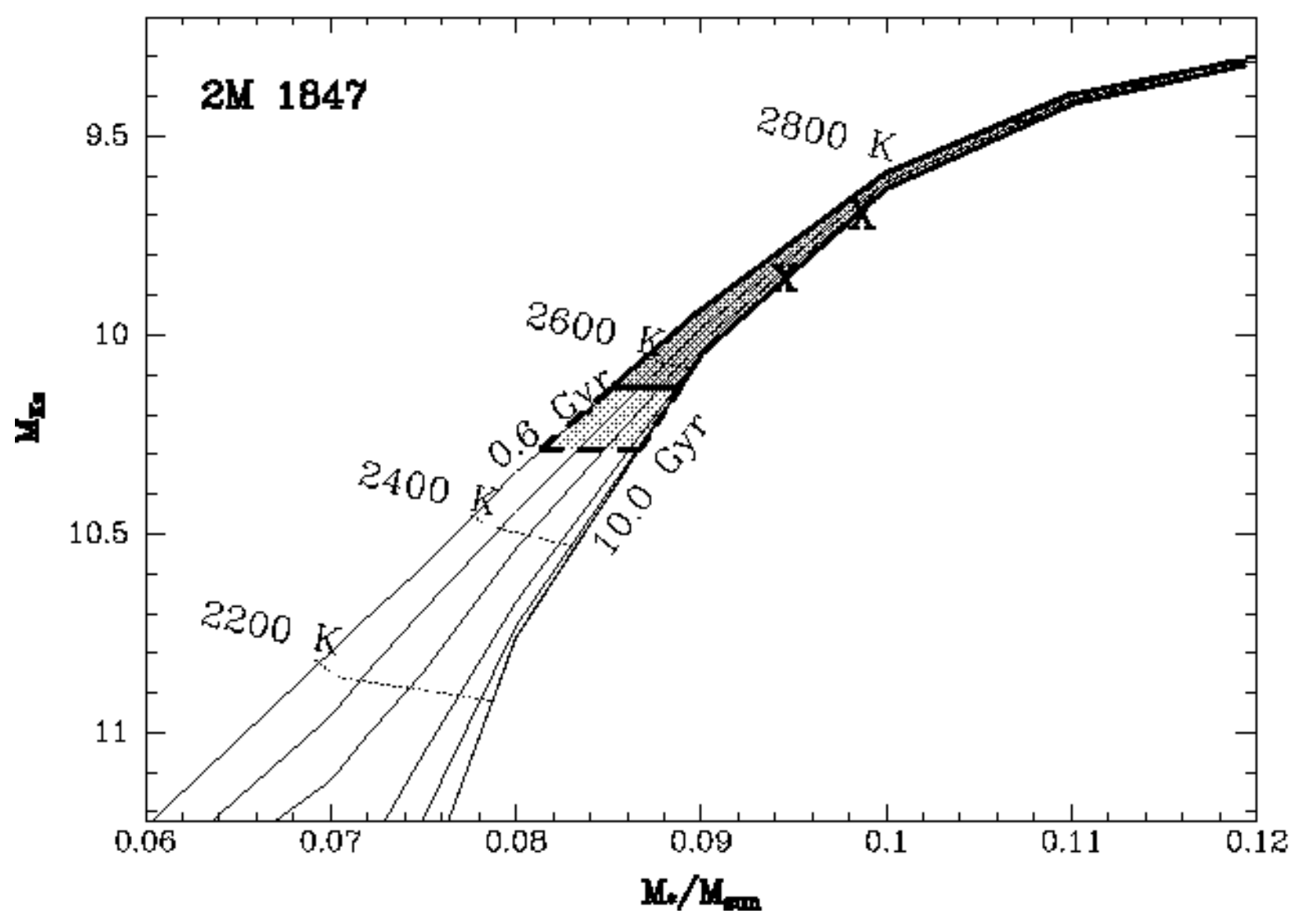

Fig. 6.- As in Figure 5, but for 2M 1847. In this case the shaded regions of uncertainty of the 2 components overlap. Hence, we outline the primary star's in solid and the companion in dotted lines (where the two regions overlap solid takes preference). The model suggests a primary mass of $0.098_{-0.012}^{+0.022} \mathrm{M}_{\odot}$ and a temperature of $2760_{-260}^{+280} \mathrm{~K}$. For the secondary the model suggests a mass of $0.094_{-0.013}^{+0.014} \mathrm{M}_{\odot}$ and temperature of $2690_{-210}^{+220} \mathrm{~K}$. 


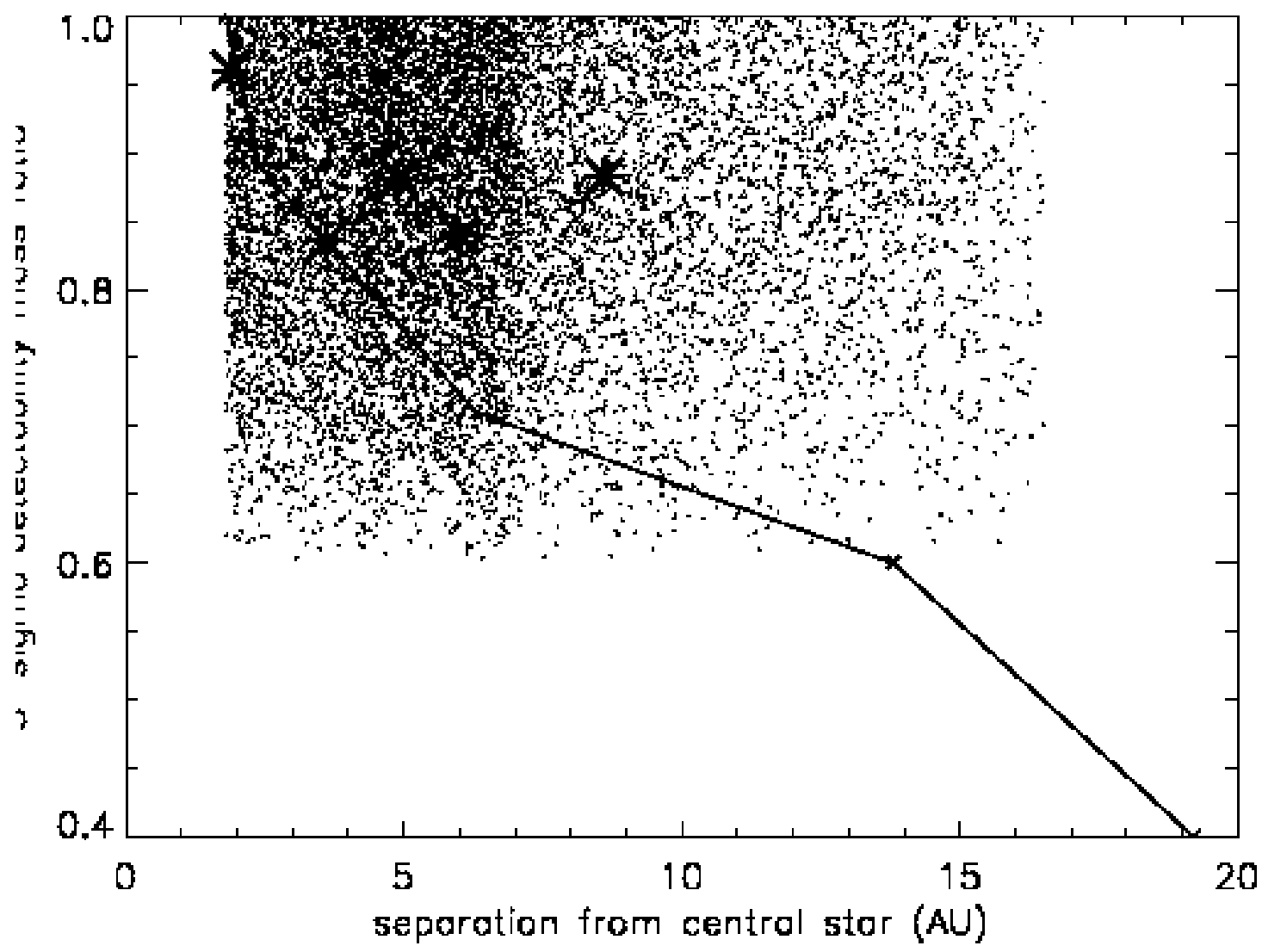

Fig. 7.- The results from a Monte Carlo simulation generating 11,670 companions distributed according to a bivariate distribution (mass ratio q and separation a) is plotted over the instrumentation sensitivity curve (connected lines). We assume the two distributions are independent. We assume a power law declining from unity to 0.6 for the mass ratio distribution (Close et al. 2003) and the profile from Figure 8 for the separation distribution for $\mathrm{a}>3 \mathrm{AU}$. The instrumentation sensitivity curve is based on modeling of a $\sim 5 \mathrm{Gyr}$ M6.5 dwarf placed at $30 \mathrm{pc}$, typical of the distances of our discovered binaries. The DUSTY models (Chabrier et al. 2000) are used to convert $\Delta H$ magnitudes to mass ratios. The 5 discovered binary systems are indicated by large asterisks. $21 \%$ of the synthetic companions fall below the instrumentation sensitivity curve but above the instrument sensitivity mass ratio cutoff of $\mathrm{q}=0.6$. This results in a sensitivity correction of 1.3 binaries. 


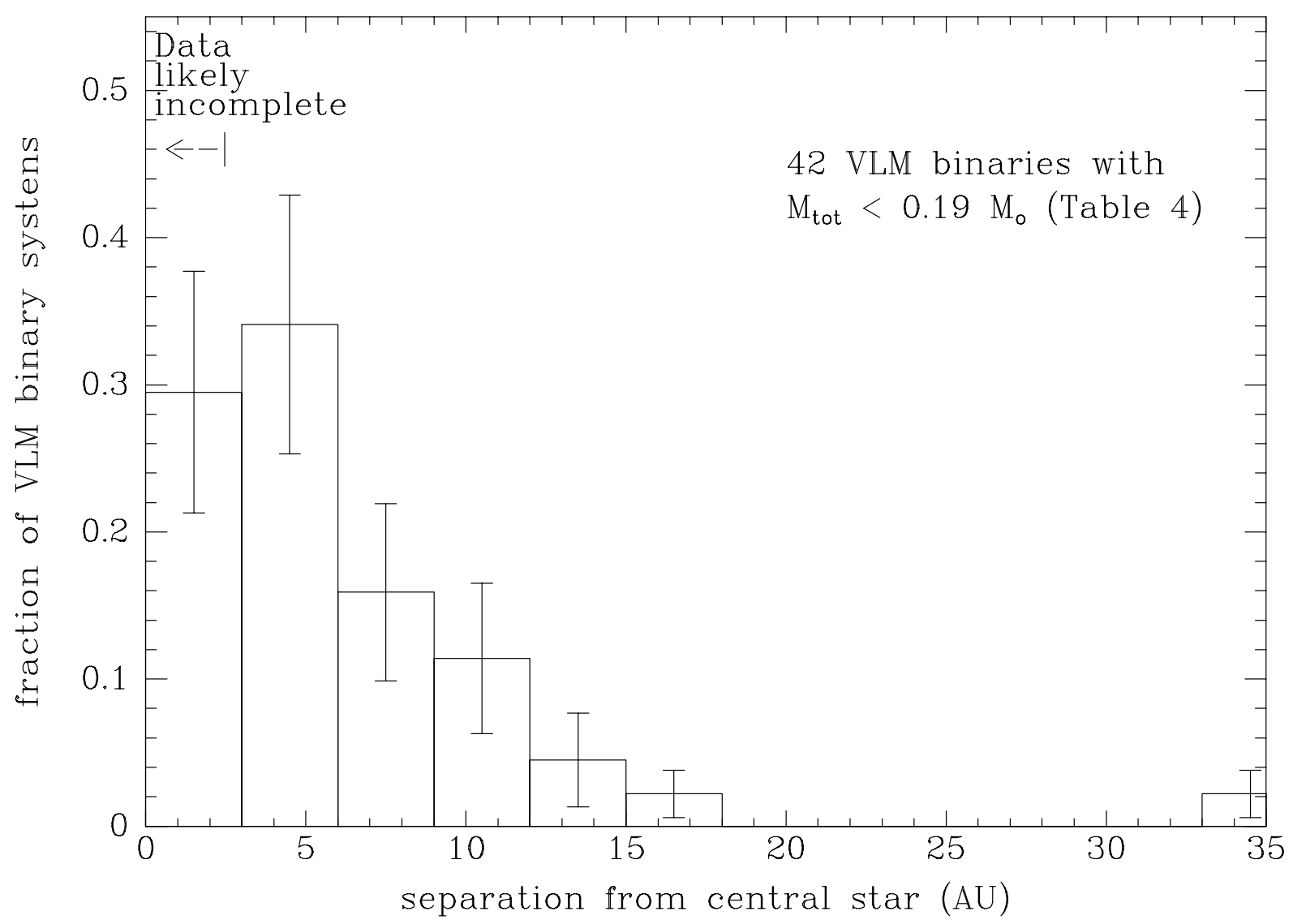

Fig. 8.- A histogram of $42 \operatorname{VLM}\left(\mathrm{M}_{t o t}<0.19 \mathrm{M}_{\odot}\right.$, component spectral types $\geq$ M6.0) binaries from Table 4 are plotted (for reasons of clarity, we leave out the 2 widest systems). The distribution is incomplete less than $\sim 3 \mathrm{AU}$; Poisson error bars are plotted. The declining profile is a real feature of the distribution as is the paucity of wide binaries greater than $15 \mathrm{AU}$. These features along with a tighter peak distribution $(\sim 3-4 \mathrm{AU})$ are significantly different from those of more massive primary stars (Fischer \& Marcy 1992; Duquennoy \& Mayor 1991). 